\title{
Neuropatía sensitiva y motora hereditaria: Enfermedad de Charcot Marie Tooth.
}

\author{
Verónica Granda-Vivanco ${ }^{1}$, Tatiana Jaramillo-Herrera ${ }^{2}$, \\ Lorena Conza González ${ }^{3}$
}

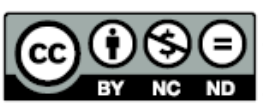

Este artículo está bajo una liencia de Creative Commons de tipo Reconocimiento - No comercial - Sin obras derivaOPEN ACCESS das 4.0 International

1 Médico General graduado en Universidad Técnica Particular de Loja, Loja- Ecuador, médico residente de emergencias del Hospital Carlos Andrade Marín. 2 Médico General graduado en Universidad Técnica Particular de Loja, Loja- Ecuador, médico residente de cirugía general del Hospital General Manuel Ygnacio Monteros.

3 Médico Internista graduado en Universidad

3 Médico Internista graduado en Universidad Hospital General Manuel Ygnacio Monteros, Médico Docente en Universidad Técnica Particular de Loja

\section{ORCID ID:}

Verónica Granda.

https:// orcid.org/0000-0002-8617-8728

Tatiana Jaramillo $\mathrm{H}$.

https://orcid.org/0000-0001-6851-2211

Lorena Conza G.

https:/ / orcid.org/0000-0002-5731-1630

Recibido: 3 - mayo - 2017

Aceptado: 29 - marzo - 2019

Publicado: 1 - noviembre - 2019

Correspondencia: Dr. Verónica Salomé

Granda Vivanco

E-mail: vero-salome@hotmail.com

Financiamiento: El presente Trabajo se declara autofinanciado por las autoras.

Conflictos de interés: Los autores declaran no poseer conflicto de interés.

\section{Resumen}

La enfermedad de Charcot Marie Tooth (ECMT) o neuropatía sensitiva y motora hereditaria es el grupo de trastornos degenerativos más común del sistema nervioso periférico, asociado a un conjunto de alteraciones genéticas que cambia la estructura, formación y mantenimiento de la mielina. Afecta a 1 de cada 2500 personas sin guardar relación con la edad, género o etnia; su etiología es únicamente genética. Según la velocidad de conducción nerviosa se clasifica en desmielinizante o CMT1, axonal o CMT2 e intermedia la misma que posee características de los dos anteriores. La ECMT en la mayoría de los casos es una enfermedad lentamente progresiva, se presenta con signos característicos de pie cavo, pierna de cigüeña, atrofia y disminución de la fuerza muscular, alteración en la percepción de estímulos vibratorios, de comienzo distal con progresión a proximal; arreflexia y alteración de la marcha. El diagnóstico se realiza en base a los antecedentes familiares, clínica y examen físico, complementando con estudios electromiográficos para determinar su clasificación. Las pruebas genéticas se deben realizar dependiendo al tipo de ECMT en sospecha, las mismas que servirán para realizar consejería familiar. En la actualidad no existe tratamiento específico ni curativo, por lo que se debe brindar apoyo con terapia física y de rehabilitación, psicológica, ocupacional, así como un manejo óptimo del dolor.

Palabras clave: Neuropatías hereditarias, Enfermedad de Charcot Marie Tooth, diagnóstico, tratamiento, revisión, Enfermedad degenerativa.
Forma de citar este artículo: Granda V Jaramillo T, Conza L. Neuropatía sensitiva y motora hereditaria: Enfermedad de Charcot Marie Tooth. Rev Med Vozandes. 2019; 30 (1): 27 - 36

\section{Abstract}

Hereditary Motor and Sensory Neuropathy: Charcot Marie Tooth disease

Charcot Mariet Tooth's disease (ECMT) or hereditary sensory and motor neuropathy is the one of the most common group of degenerative disorders of the peripheral nervous system, related with a set of genetic alterations that changes the structure, formation and maintenance of myelin. It affects 1 out of 2500 people without considering the age, gender or ethnicity; its etiology is entirely genetic. According to the nerve conduction velocity it is classified in demyelinating or CMT1, axonal or CMT2 and intermediate the same that has the features of the two previous ones. ECMT in majority of cases it is a slowly progressive disease, presenting with characteristic signs of high instep, stork leg, atrophy and decreased muscle strength, altered perception of vibratory stimuli, distal beginning with proximal progression; arreflexia and alteration of march. The diagnosis is based on family history, clinical and physical examination, complemented by electromyography studies, to determine their classification. Genetic tests are taken, based on the type of suspected ECMT, and these will be used for family counseling. Nowadays there is no specific and curative treatment, that's why support should be provided with physical and rehabilitation therapies, psychological, occupational, as well as an optimal pain control. 


\section{Introducción}

La enfermedad de Charcot Marie Tooth, recibe su nombre en honor a los tres médicos que la describieron por primera vez en 1886, JeanMartin Charcot, Pierre Marie y Howard Henry Tooth, bajo el título de "Una forma particular de atrofia muscular progresiva". Ellos describieron el síndrome en cinco pacientes, dos de ellos hermanos, con inicio en la infancia y adolescencia, que presentaban características similares. ${ }^{(1)}$

La enfermedad de Charcot Marie Tooth (ECMT) o Neuropatía sensitiva y motora hereditaria (NSMH), es el grupo de trastornos degenerativos, más común del sistema nervioso periférico, está asociada a un conjunto de alteraciones genéticas que cambia la estructura, formación y mantenimiento de la mielina ${ }^{(2)}$; es una condición genéticamente heterogénea, con más de 75 genes involucrados ${ }^{(3)}$.

Siendo la enfermedad neurológica hereditaria más común, progresiva, incapacitante, con diagnóstico tardío, limitado manejo y escasa información, actualmente, se considera infradiagnosticada, razón por la cual se realiza la siguiente revisión como una herramienta para su oportuna detección y manejo.

\section{Epidemiología}

La ECMT afecta a 1 de cada 2.500 personas en Estados Unidos (3), en España tiene una prevalencia de 28,2 casos por cada 100.000 habitantes ${ }^{(4)}$. Esta patología no guarda relación con la edad, género y etnia, aunque se considera que su inicio empieza en la niñez y adolescencia ${ }^{(4)}$.

Tabla 1. Clasificación genética de Charcot Marie Tooth
El $70 \%$ de todos los casos de ECMT es de tipo desmielinizante (CMT1) con una prevalencia de 1 por cada 5 mil personas. El subtipo más común es (CMT1A) que representa el $50 \%$ de todos los casos, seguido del subtipo CMTIX. ${ }^{(3)}$

Existe un subtipo especial que se denomina Neuropatía hereditaria con susceptibilidad a la parálisis por presión, se presenta con 16 casos por cada 100.000 habitantes, pero se considera que este subtipo por sus características clínicas se encuentra infradiagnosticado ${ }^{(3)}$.

Existe una fuga de información de casos ya que no se encuentra un CIE10 específico para esta patología, según la clasificación el código que más se acerca por sus características es el G60.0 (polineuropatía hereditaria idiopática), en el 2013 en Ecuador se encuentran 19 casos dentro de este código ${ }^{(5)}$, sin embargo no se puede especificar cuántos de ellos pertenecen a ECMT por lo que no se encuentra información al respecto en epidemiologia.

\section{Clasificación}

De acuerdo al estudio de conducción nerviosa, se puede clasificar:

- Desmielinizante o CMT1 (velocidad de conducción <38 m/segundo en nervio ulnar).

- Axonal o CMT2 (conducción reducida, con amplitudes $>38 \mathrm{~m} /$ segundo).

- Intermedio (desmielinizante y axonal). ${ }^{(2)}$

Cada una de ellas así mismo presenta subtipos que se detallan en la tabla $1^{(6)}$

\begin{tabular}{|c|c|c|c|c|}
\hline \multicolumn{2}{|l|}{ Tipo } & \multirow{2}{*}{$\begin{array}{l}\text { Subtipo } \\
\text { CMTIA }\end{array}$} & \multirow{2}{*}{$\begin{array}{l}\text { Genes } \\
\text { PMP22 }\end{array}$} & \multirow{2}{*}{$\begin{array}{l}\text { Clínica } \\
\text { Forma clásica: Déficit motor de forma lenta y progresiva, } \\
\text { presencia de pie cavo, ausencia de síntomas sensitivos. }{ }^{(7)} \\
\text {-Inicio a cualquier edad. }\end{array}$} \\
\hline \multirow[t]{8}{*}{$\begin{array}{l}\text { Autosómico } \\
\text { dominante }\end{array}$} & \multirow[t]{8}{*}{$\begin{array}{l}\text { Desmielinizante } \\
\text { CMT1 }\end{array}$} & & & \\
\hline & & HNPP & PMP22 & $\begin{array}{l}\text { Mononeuropatía con debilidad muscular indolora con al } \\
\text { menos un episodio de parálisis nerviosa aguda. (8) -Inicio en } \\
\text { la adolescencia con mayor expresión en la adultez. }\end{array}$ \\
\hline & & CMTIB & MPZ & Se presenta de forma clasica. (9) -Inicio $1^{\circ}-2^{\circ}$ década. \\
\hline & & CMTIC & LITAF & $\begin{array}{l}\text { Debilidad distal con reflejos nerviosos reducidos. (9) -Inico } \\
\text { en la infancia. }\end{array}$ \\
\hline & & CMTID & EGR2 & $\begin{array}{l}\text { Debilidad distal con reflejos ausentes }{ }^{(9)} \text {. - Inicio en la } 1^{\circ} \\
\text { década. }\end{array}$ \\
\hline & & CMTIE & PMP22 & $\begin{array}{l}\text { Discapacidad clinica severa con afectacion o perdida ves- } \\
\text { tibular y/o nervio craneal. }{ }^{\left({ }^{(0)}\right.} \text {-Inicio en la infancia. }\end{array}$ \\
\hline & & CMTIF & NEFL & $\begin{array}{l}\text { Forma clásica sereva con retraso en el desarrollo motor. (11) } \\
\text {-Inicio } 1 \text { - } 13 \text { años. }\end{array}$ \\
\hline & & $\begin{array}{l}\text { CMT } \\
\text { "PLUS" }\end{array}$ & FBLN5 & $\begin{array}{l}\text { Hiperelasticidad de la piel, degeneración macular relacio- } \\
\text { nada con la edad. }{ }^{(6)} \text {-Inicio } 4^{\circ}-5^{\circ} \text { década. }\end{array}$ \\
\hline
\end{tabular}


Tipo

Autosómico Axonal dominante CMT2
Subtipo Genes Clínica

CMT2A MFN2

CMT2B RAB7

CMT2C TRPV4

CMT2D GARS

CMT2E NEFL

CMT2F HSPBI

CMT2G 12q12q13.2

CMT2I - MPZ

CMT2J

CMT2K GDAP1

CMT2L HSPB8

CMT2M DNM2

CMT2N AARS

CMT2O DYNCIHI

CMT2P LRSAMI

CMT2Q DHTKDI

HMSN-P TFG años.
Debilidad distal con reflejos ausentes a nivel distal. ${ }^{(9)}$-Inicio a los 10 años.

Perdida sensitiva y debilidad distal (9). -Inicio en la $2^{\circ}$ década.

Debilidad distal y paralisis de cuerdas vocales, reflejos ausentes. ${ }^{(9)}$ -Inicio en la $1^{\circ}$ década.

Debilidad distal y reflejos reducidos (9). -Inicio 16 - 30 años .

Debilidad distal y reflejos reducidos ${ }^{(9)}$. Inicio $1^{\circ}-5^{\circ}$ década.

Dificultad para caminar con reflejos reducidos ${ }^{(9)}$. -Inicio 6 a 54

Debilidad distal con reflejos reducidos ${ }^{(9)}$. -Inicio de 15 a 25 años.

Forma clásica con sordera y alteraciones pupilares (pupila de Adie) (11). -Inicio en el adulto mayor.

Paralisis progresiva de la cuerda vocal (11). -Inicio variable.

Debilidad distal con reflejos reducidos ${ }^{(9)}$. Inicio de 15 - 33 años.

Debilidad distal con reflejos reducidos ${ }^{(9)}$. Inicio de 0 a 50 años.

Debilidad distal con reflejos reducidos ${ }^{(9)}$. Inicio de 6 a 54 años.

Dificultades en el aprendizaje. ${ }^{(6)}$ Inicio infancia temprana.

Temblores y asimetría ${ }^{(6)}$-Inicio 27 - 40 años.

Forma clásica. Inicio de 13 - 25 años.

Debilidad proximal, presencia de calambres con reflejos ausentes ${ }^{(9)}$. -Inicio 17 - 50 años.
Tipo

Autosómico recesivo
Subtipo

AR-CMTIA

$A R-C M T 1 B 1$

AR-CMTIB2

AR-CMTIB3

AR-CMTIC

AR-CMTID

AR-CMTIE

AR-CMTIF

AR-CMTIG

AR-CMTIH

AR-CMT1

\section{Genes Clínica}

GDAP1 Debilidad distal y reflejos reducidos ${ }^{(9)}$. Inicio $<2$ años.

MTMR2 Debilidad distal y proximal con reflejos ausentes ${ }^{(9)}$ |nicio de 2 - 4 años.

MTMR13 Forma clásica severa con glaucoma y cifoescoliosis. (6) Inicio 4 - 5 años.

MTMR5 Pie Plano con escoliosis. (6) Inicio de $5-11$ años.

SH3TC2 Retardo al caminar, reflejos reducidos. (9) Inicio temprano $1^{\circ}-2^{\circ}$ década.

NDRG1 Alteraciones en la marcha, reflejos ausentes ${ }^{(9)}$ Inicio 1- 10 años.

EGR2 Hipotonía y reflejos ausentes ${ }^{(9)}$ Inicio al nacimiento.

PRX

Retardo al caminar y reflejos ausentes (9) Inicio 1 - 3 años.

HK1 Debilidad muscular distal con pérdida sensorial (11) Inicio 8 - 16 años.

FGD4

Retardo al caminar y reflejos ausentes ${ }^{(9)}$. Inicio $10-24$ meses.

Trastorno en la coordinación con discapacidad severa. Inicio Infancia 


\section{Tipo}

\section{Autosómico} recesivo
Subtipo

Axonal CMT2
AR-CMT2A

AR-CMT2B

AR-CMT2C NEFL

AR-CMT2F HSPB1

AR-CMT2K GDAPI

AR-CMT2P

HMSN VI

ARAN-NM

GAN

MFN2

GAN

\section{Genes Clínica}

Debilidad distal, reflejos reducidos. ${ }^{(9)}$-Inicio $2^{\circ}$ década.

Debilidad distal, reflejos ausentes en zona distal. ${ }^{(9)}$ - Inicio 28 - 42 años.

Reflejos ausentes a nivel de tobillo, rótula y miembros superiores. (11) -Inicio $1^{\circ}$ década.

Debilidad muscular de miembros inferiores ${ }^{(6)}$-Inicio variable.

Parálisis de las cuerdas vocales y deformidades del esqueleto ${ }^{(6)}$. -Inicio temprano.

LRSAMI Disfunción eréctil . Inicio en la $3^{\circ}-4^{\circ}$ década. ${ }^{(6)}$

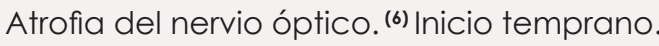

Neuromiotonia. ${ }^{(6)}$-Inicio en la $1^{\circ}$ década.

Enfermedad severa con compromiso del SNC. ${ }^{\left({ }^{6}\right)}$-Inicio en la infancia.

\begin{tabular}{|c|c|c|c|c|}
\hline Tipo & & Subtipo & Genes & Clínica \\
\hline \multirow{4}{*}{$\begin{array}{l}\text { Ligado a } \\
\text { cromosoma } \\
X\end{array}$} & intermedio & CMTX1 & GJB 1 & $\begin{array}{l}\text { Debilidad distal con reflejos ausentes }{ }^{(9)} \text {. -Inicio } 1^{\circ}{ }^{\circ}{ }^{\circ} \\
\text { década. }\end{array}$ \\
\hline & & CMTX4 & AlFM1 & $\begin{array}{l}\text { Sordera con retraso mental del } 60 \% .{ }^{(6)} \text {-Infancia tempra- } \\
\text { na. }\end{array}$ \\
\hline & & CMTX5 & PRPS 1 & $\begin{array}{l}\text { Pérdida de la audición con neuropatía optica. }{ }^{(6)} \text { Inicio en } \\
\text { la infancia. }\end{array}$ \\
\hline & & CMTX6 & PDK3 & Forma clasica. ${ }^{(6)}$ Inicio en la infancia. \\
\hline
\end{tabular}

Tipo

\section{Dominancia} intermedia
Subtipo

Intermedio

DI-CMTA

DI-CMTB

DI-CMTC

DI-CMTD

DI-CMTE

DI-CMTF 


\section{Características clínicas y patológicas de los tipos de ECMT}

-ECMT autosómico dominante.- se considera que es la forma más frecuente de esta patología, se subdividen en:

- CMT1.- se caracterizan por presentar una velocidad de conducción inferior a $38 \mathrm{~m} \backslash \mathrm{s}$ y a nivel de patológico presentan disminución de la mielinización de axones y la presencia de bulbos de cebolla, compuestos por varias capas de lámina basal, tejido conectivo y células de Shwann, la mayoría de pacientes con este subtipo suelen presentarse clínicamente con una discapacidad de leve a moderada, aunque existen algunos casos con marcada disminución de sus capacidades motoras que incluso llegan a necesitar silla de ruedas. La edad de inicio en la presentación de esta enfermedad es variable con relación al gen afectado en cada subtipo. (Ver tabla 1) (6)

- CMT2.- representa $1 \backslash 3$ de los casos autosómicos dominantes, este subtipo suele presentarse con velocidades de conducción dentro de parámetros normales pero su amplitud puede estar ausente o disminuida. En la biopsia suele encontrarse pérdida axonal con signos de regeneración, se presenta clínicamente de forma clásica, que se verá con más detalle en la sección "curso clínico", su rango de inicio es más impredecible y depende mucho de su variabilidad genética. En casos especiales (subtipo CMT2A) se presentan manifestaciones atípicas como: signo de Babinski, hiperreflexia y atrofia óptica. (6)

- ECMT autosómico recesivo. - son casos extremadamente raros, se presentan en edades tempranas y su nivel de progresión es rápida, ocasionando que la presencia de deformidades sea marcada. Se subdividen al igual que en la autosómica dominante en CMT1 y CMT2, a nivel patológico se han encontrado lesiones desmielinizantes destacadas que producen una lesión axonal secundaria junto a lesiones de la mielina específicas de cada subtipo. ${ }^{(6)}$

- ECMT ligado al cromosoma X.- las mujeres que presentan este subtipo tienen una probabilidad de transmisión del $50 \%$ y en los hombres la probabilidad asciende a un $100 \%$ (3). Hasta el momento solo se han identificado 4 subtipos (véase en la tabla1), las velocidades de conducción nerviosa suelen encontrarse en un rango intermedio de 25-40 m\s, algunos subtipos suelen presentar síntomas especiales como sordera leve y atrofia óptica. ${ }^{(6)}$

- ECMT dominante intermedio. - presenta un fenotipo clásico, con una intensidad leve a moderadamente severa, suelen presentarse con una velocidad de conducción de 25-50 m\s. Patológicamente se ha encontrado una acumulación anormal de $\beta$-actina en el citoplasma de las células de Shwann, se han identificado 6 subtipos clínicos, suelen presentarse con manifestaciones clínicas especiales como: proteinuria y glomeruloesclerosis. ${ }^{(6)}$

\section{Factores de riesgo}

No existe evidencia de factores de riesgo conocidos para la enfermedad, excepto una historia familiar de ECMT, pero la ausencia de este factor no descarta el diagnóstico. El riesgo de transmisión genética de la enfermedad depende del subtipo de ECMT. ${ }^{(3)}$
En las formas dominantes más comunes (CMT1 y CMT2), un antecedente de primer grado es suficiente para que aumente la predisposición genética y cada hijo tiene un $50 \%$ de probabilidades de heredar el trastorno. En contraste con las formas recesivas tienen una probabilidad de un $25 \%$ de que los hijos tengan el riesgo de padecer la enfermedad. (12)

Las mujeres con una forma ligada al cromosoma $X$ de CMT tienen un $50 \%$ de probabilidad de transmisión de la enfermedad, mientras que los hombres con una forma ligada al cromosoma $X$ tienen un $100 \%$ de probabilidad de transmitir la enfermedad a sus hijas $y$ $0 \%$ de probabilidad de transmitirla a sus hijos. ${ }^{(3)}$

\section{Diagnóstico}

Para realizar un óptimo diagnóstico es importante una historia clínica minuciosa recalcando la historia familiar, un examen físico neurológico completo y estructurado.

La presencia de neuropatía periférica, con una historia familiar positiva y pruebas genéticas confirmatorias son características del diagnóstico de CMT. Cuando estas pruebas son negativas, y el curso clínico está en etapa subaguda comúnmente es difícil distinguir la ECMT de una neuropatía inflamatoria adquirida, por lo cual puede ser necesario realizar una biopsia del nervio sural para detectar la presencia de signos de inflamación y descartar ECMT. ${ }^{(3)}$

\section{Curso clínico}

Los signos y síntomas en la ECMT son específicos de acuerdo al subtipo y la alteración genética, pero entre los síntomas generales se encuentran:

- Antecedentes familiares de la neuropatía con pie cavo o alteración de la marcha ${ }^{(3)}$.

- La afectación es simétrica, aunque en raras ocasiones puede ser asimétrica ${ }^{(3)}$.

- Las características más comunes son debilidad y atrofia de la pierna (pierna de cigüeña) y el pie (pie cavo), dedos en forma de martillo ${ }^{(12)}$.

- Alteración del equilibrio y la marcha, en las fases tempranas la evaluación de la marcha revela pérdida del patrón talón-punta seguido de la caminata en puntillas (13)

- Arcos del pie altos son comunes, pero no patognomónicos. Si el pie cavo se asocia con arreflexia, la probabilidad de CMT es alta ${ }^{(3)}$.

- Hiporreflexia tendinosa profunda o arreflexia (3).

- Fuerza muscular reducida asociada a atrofia, afectando a los músculos distales en primer lugar, manteniéndose conservados en las partes más proximales ${ }^{(12)}$.

- Disminución de la sensibilidad en especial a los estímulos vibratorios aunque también puede afectar a disminución de percepción del dolor, temperatura y propiocepción. ${ }^{(13)}$

- Deformidades en miembros superiores (mano en garra) (13) 


\section{Diagnósticos diferenciales}

\section{Tabla 2: Diagnósticos diferenciales de la ECMT}

\section{Patología}

Neuropatía Diabética

Polineuropatía desmielinizante inflamatoria crónica.

Neuropatía periférica adquirida

Paraplejia espástica hereditaria

\section{Características clínicas}

Es más común en adultos. Presenta:

- Pérdida de la sensibilidad vibratoria y alteración de propiocepción refleja.

- Deterioro de la sensibilidad al dolor, tacto, luz y la temperatura

- Disminución o ausencia de reflejos del tobillo que ocurren temprano en la enfermedad. ${ }^{(14)}$

- Se puede urilizar el screening de Michigan para identificar la clinica de la neuropatia y evaluar su severidad con una puntuación compuesta por clínica y conduccion nerviosa ${ }^{(15)}$

- Simétrica con mayor implicación motora que sensitiva.

- Afecta tanto los músculos distales y proximales.

- Curso lentamente progresivo.

- Disminución o ausencia global de reflejos.

- Deterioro sensorial para la vibración y el sentido de posición. ${ }^{(16)}$

Las causas incluyen toxinas, hipotiroidismo, Deficiencia de vitaminas e insuficiencia renal.

- Presentación aguda en exposición a tóxicos o condiciones inflamatorias.

- Reflejos tendinosos profundos están difusamente ausentes.

- Simétrica afectación bilateral, mayormente distal.

- Síntomas autonómicos: diaforésis, arritmias cardíacas, hipotensión o disfunción del intestino y la vejiga ${ }^{(17)}$

La espasticidad, hiperreflexia, y los signos de Babinski son indicativos de una lesión de la motoneurona superior.

Varios patrones de progresión como:

- curso relativamente no progresivo,

- empeoramiento progresivo que se estabiliza con el tiempo

- inexorable declive. (18)

Degeneración espinocerebelosa
- Ataxia en ocasiones con neuropatía asociada.

- hiperreflexia,

- aumento del tono,

- respuestas plantares extensoras

\section{Exámenes complementarios}

Electromiografía

Biopsia del nervio sural: puede mostrar la inflamación, además de desmielinización.

Dependen de la causa subyacente.
- Pruebas genéticas: mutación genética específica. Entre las más comunes mutaciones del SPG 1 al SPG7.

- RM: atrofia de la médula espinal, posible atrofia de la corteza cerebral.

RM: evidente degeneración espinocerebelosa.

*Principales patologías que nos permiten realizar un Diagnóstico diferencial con la enfermedad de Charcot Marie Tooth en base a la clínica relevante y pruebas complementarias.

†RM: Resonancia magnética. 


\section{Estudios de conducción nerviosa (NCS)}

Una vez diagnosticado es necesario realizar el estudio de conducción nerviosa para poder determinar si es motor, sensitivo o mixto. Además, determina si afecta a la mielina y la célula de Schwann, o el axón. ${ }^{(3)}$

En el CMTl (incluyendo CMTIA) está asociada con velocidades de conducción se encuentran reducidas o ausentes (15 a $38 \mathrm{~m} /$ segundo), mientras que en CMT2 (incluyendo CMT2A) se asocia con amplitudes reducidas, pero con velocidades de conducción relativamente normales de $>38 \mathrm{~m} /$ segundo (3) y la intermedia con un velocidad de conducción de 25 y $45 \mathrm{~mm} / \mathrm{s}$, todas estas mediciones realizadas en el nervio mediano ${ }^{(4)}$

\section{Pruebas genéticas}

La clasificación genética no es necesaria para realizar un diagnóstico, las pruebas genéticas deben realizarse para brindar al paciente un consejo genético adecuado, para tomar decisiones referentes a planificación familiar conociendo más ampliamente la enfermedad. (3) También se debe tener presente los aspectos éticos, legales y psicosociales que conllevan estos estudios en niños y adolescentes, previo a realizarlos.

Se debe recalcar que estas pruebas son de alto costo y que al momento únicamente existen contados laboratorios de genética en nuestro país, razón por la que se sugiere que para la solicitud de este examen se considere la clínica y los resultados de electrofisiología (Véase tabla 1), así a continuación unos ejemplos:

- Pacientes con sintomatología clásica, sin retraso en el inicio de la marcha, con herencia autosomica dominante se sospecha de CMT1A, la prueba genética a realizar es la PMP22, si ésta es negativa se procederá a solicitar la GBJ1 y MPZ (4)

- Cuando se encuentra el cuadro clinico anterior pero la velocidad de conduccion muy lentificada $<15 \mathrm{~mm} \backslash \mathrm{s}$, se debe realizar en primera instancia el gen MPZ o PMP22 ${ }^{(4)}$

\section{Valoración de progresión de CMT}

Para valorar la gravedad existe una escala denominada CMTNSv2 (tabla 3) con un total de 36 puntos que incluyen los hallazgos clínicos y electrofisiología.

Se clasifican sus resultados de: 0-10 puntos leve deterioro, 11-20 puntos moderado, $y \geq 21$ puntos deterioro grave. ${ }^{(19)}$

\section{Tratamiento}

La ECMT no tiene un tratamiento curativo, unicamente es de apoyo, se puede recomendar una cirugía ortopédica para lograr en el paciente una alineación adecuada de la posición neutral, aumento en la movilidad e inclusive a menudo los pacientes mejoran su postura y pueden realizar la marcha, aunque se debe recordar que la enfermedad es de carácter progresivo y que la cirugía no siempre es correctiva, por lo que otra opción es el uso de ayudas técnicas, ortopédicas que le permitan la deambulación. (3)

\section{Terapeutica no farmacológica: -Atención psicológica}

Una revisión sistemática con metodología adecuada valoró los indicadores psiquiátricos y la calidad de vida en los pacientes con ECMT, concluyó que estos pacientes tienen mayor tendencia a desarrollar síntomas depresivos que la población en general y un mayor riesgo de reducción en la calidad de vida con deterioro significativo del sueño. ${ }^{(20)}$

Para muchos padres y familiares el convivir con esta patologia produce niveles altos estrés psicosocial que conlleva un aislamiento social, depresión y ansiedad, lo que afecta parcialmente al paciente para su acceso a las actividades sociales, su proceso de adaptación y aceptación de la enfermedad. Es por ello que la evaluación, atención, seguimeinto psiquiátrico y psicológico son fundamentales.

\section{-Fisioterapia y ejercicio}

Un Estudio publicado en Pubmed sobre el papel de la rehabilitación en el manejo de la enfermedad de Charcot Marie Tooth, que incluyó a pacientes con signos y síntomas de debilidad y atrofia en la parte distal de las extremidades, parestesias, contracturas de los tobillos y dificultad para caminar, realizaron evaluaciones como examen clínico, índice de Barthel, tiempo UP AND GO en prueba, medición de la autonomía de los movimientos de tobillo y pruebas manuales musculares para evaluar la mejoría de la enfermedad, con estos pacientes se realizaron ejercicios de rehabilitación como: terapia ocupacional, montar bicicleta estática, corriente Galvánica, ejercicios en agua y órtesis de tobillo y pie, concluyendo que la terapia aplicada no tuvo cambios significativos sobre el estado neurológico clínico de los pacientes pero proporciona cierta mejoría en la contractura de tobillo con una mejor movilidad y estabilidad en la marcha. ${ }^{(21)}$

Un estudio informa los cambios físicos y emocionales inducidos por la actividad deportiva de un nadador paraolímpico con ECMT, en el cual compararon las evaluaciones antes y después de iniciar la actividad deportiva por un periodo de 5 años encontrando mejoría en un aumento de los músculos proximales de las extremidades superiores, mayor capacidad de impulsar la silla de ruedas, mejoría en la calidad de vida, reducción de la ansiedad, depresión y mejora de autoestima. ${ }^{(22)}$

Un artículo publicado en PubMed sobre los efectos del ejercicio autoseleccionado en los subtipos de ECMT con una cantidad de 297 pacientes, demostró que los participantes con CMTl y 2 que se ejercitaron son significativamente más fuertes al realizar la flexión del codo y dorsiflexión de los que no lo hicieron, disminuyendo la discapacidad en esta población. (23) 
Tabla 3. Escala para valorar la gravedad y progresión del CMT (CMTNSv2) (19)

\begin{tabular}{|c|c|c|c|c|c|c|}
\hline \multicolumn{2}{|c|}{ Parámetro } & 0 & 1 & 2 & 3 & 4 \\
\hline \multicolumn{2}{|c|}{ Síntomas sensoriales } & Ninguno & $\begin{array}{l}\text { Ubicados en } \\
\text { los huesos } \\
\text { del tobillo }\end{array}$ & $\begin{array}{l}\text { Hasta la mitad } \\
\text { distal de la } \\
\text { pantorrilla }\end{array}$ & $\begin{array}{l}\text { Hasta la mitad } \\
\text { proximal de la } \\
\text { pantorrilla, inclu- } \\
\text { yendo la rodilla }\end{array}$ & $\begin{array}{l}\text { Por encima de } \\
\text { la rodilla }\end{array}$ \\
\hline \multicolumn{2}{|c|}{ Los síntomas motores (piernas) } & Ninguno & $\begin{array}{l}\text { Pérdida del } \\
\text { patrón talón- } \\
\text { tobillo }\end{array}$ & $\begin{array}{l}\text { Soporte para } \\
\text { el tobillo o la } \\
\text { estabilización }\end{array}$ & $\begin{array}{l}\text { Ayudas para } \\
\text { caminar (bastón, } \\
\text { andador) }\end{array}$ & Silla de ruedas \\
\hline \multicolumn{2}{|c|}{ Los síntomas motores (brazos) } & Ninguno & $\begin{array}{l}\text { Dificultad } \\
\text { leve con } \\
\text { botones }\end{array}$ & $\begin{array}{l}\text { Tiene dificultad } \\
\text { severa (inca- } \\
\text { pacidad de } \\
\text { abotonarse) }\end{array}$ & $\begin{array}{l}\text { Incapaz de cortar } \\
\text { la mayoría de los } \\
\text { alimentos }\end{array}$ & $\begin{array}{l}\text { Debilidad pro- } \\
\text { ximal }\end{array}$ \\
\hline \multicolumn{2}{|c|}{ Sensibilidad (Pinchazo) } & Normal & $\begin{array}{l}\text { Disminuido } \\
\text { por debajo o } \\
\text { por lo huesos } \\
\text { del tobillo }\end{array}$ & $\begin{array}{l}\text { La disminución } \\
\text { de hasta la } \\
\text { mitad distal de } \\
\text { la pantorrilla }\end{array}$ & $\begin{array}{l}\text { La disminución } \\
\text { de hasta la mitad } \\
\text { proximal de la } \\
\text { pantorrilla, Inclu- } \\
\text { yendo la rodilla }\end{array}$ & $\begin{array}{l}\text { Disminución } \\
\text { por encima de } \\
\text { la rodilla }\end{array}$ \\
\hline \multicolumn{2}{|l|}{ Vibración } & Normal & $\begin{array}{l}\text { Reducido en } \\
\text { dedo pulgar } \\
\text { del pie }\end{array}$ & $\begin{array}{l}\text { Reducción en } \\
\text { el tobillo }\end{array}$ & $\begin{array}{l}\text { Reducción en la } \\
\text { rodilla (tibial tube- } \\
\text { rosidad) }\end{array}$ & $\begin{array}{l}\text { Ausente en } \\
\text { la rodilla y el } \\
\text { tobillo }\end{array}$ \\
\hline \multicolumn{2}{|c|}{ Fuerza (piernas) } & Normal & $\begin{array}{l}>4 \text { en la } \\
\text { flexión dorsal } \\
\text { del pie }\end{array}$ & $\begin{array}{l}\leq 3 \text { en pie (dor- } \\
\text { siflexión) o } \leq 3 \\
\text { en pie (flexión } \\
\text { plantar) }\end{array}$ & $\begin{array}{l}\leq 3 \text { a pie dorsi- } \\
\text { flexión }+\leq 3 \text { en } \\
\text { flexión plantar }\end{array}$ & $\begin{array}{l}\text { Debilidad pro- } \\
\text { ximal }\end{array}$ \\
\hline \multicolumn{2}{|c|}{ Fuerza (brazos) } & Normal & $\begin{array}{l}\text { >4 en intrín- } \\
\text { secos de la } \\
\text { mano }\end{array}$ & $\begin{array}{l}\leq 3 \text { en intrín- } \\
\text { secos mano } \\
\text { músculos }\end{array}$ & $\begin{array}{l}\leq 5 \text { en la muñeca } \\
\text { (extensores) }\end{array}$ & $\begin{array}{l}\text { Débil por enci- } \\
\text { ma del codo }\end{array}$ \\
\hline \multirow{3}{*}{$\begin{array}{l}\text { Electro- } \\
\text { miografía } \\
\text { CMAP }\end{array}$} & Nervio Cubital & $\geq 6 \mathrm{mV}$ & $4-5,9 \mathrm{mV}$ & $2-3,9 \mathrm{mV}$ & 0,1 a $1,9 \mathrm{mV}$ & Ausente \\
\hline & Nervio Mediano & $\geq 4 \mathrm{mV}$ & 02.08 a 03.09 & 1.2 a 2.7 & $0,1-1,1$ & Ausente \\
\hline & Nervio Radial & $\geq 15 \mathrm{mV}$ & 10 a $14,9 \mathrm{mV}$ & $5-9,9 \mathrm{mV}$ & $1-4,9 \mathrm{mV}$ & $<1 \mathrm{mV}$ \\
\hline
\end{tabular}

En los pacientes con ECMT las contracturas musculares más frecuentes son: banda iliotibial, isquiotibiales, flexores de cadera, soleo y gemelos, aductores, tibial posterior, fascia plantar, flexores de dedos ocasionando dedos en garra. ${ }^{(9)}$

Los ejercicios de fortalecimiento muscular aeróbicos moderados se debe iniciar antes de la degeneración del nervio y aumento de la debilidad muscular, las actividades de alta resistencia no son indicados por lo que se sugiere fortalecimiento regular en combinación con ejercicios acuáticos como actividad de recreación. También se recomienda estiramientos a tolerancia para prevenir el acortamiento del tendón de Aquiles; y un examen regular para evitar úlceras por presión en los pies. Si hay dolor, calambres o mioglobinuría en 24 h se debe modificar la actividad física. (9)

\section{-La terapia ocupacional}

Con la evolución de la enfermedad los pacientes pueden desarrollar contracturas en los dedos por lo que se recomienda terapias con instrumentos de escritura 0 utensilios para comida, que permita mejorar el movimiento diario.

La férula de uso nocturno se sugiere a veces, para aumentar el rango de movimiento en el tobillo, sin embargo 2 estudios clínicos ramdomizados, no encontraron beneficios estadísticamente significativos. ${ }^{(3)}$

\section{Terapéutica farmacológica -Uso de la Vitamina C}

Es importante recalcar que esta terapia aún está siendo evaluada, por lo que los resultados son discordantes.

Los estudios realizados en ratones con CMT1A han demostrado que altas dosis de ácido ascórbico (vitamina C) reducen la expresión del gen PMP22 actuando sobre los niveles de AMPC intracelular y la actividad de adenilato ciclasa ${ }^{(24)}$. Como se conoce el CMTIA es causada por un exceso de proteína PMP22 debido a la presencia de una copia extra del gen, la reducción en la expresión del mismo podría conducir a una mejora en los síntomas de CMT1A. (24)

Otro estudio clínico ramdomizado, realizado en niños con CMTIA en Australia no encontró ninguna mejora con ácido ascórbico en las velocidades de conducción nerviosa por un periodo de más de 1 año, y estudios europeos han tenido resultados similares ${ }^{(25)}$, Otro estudio 
realizado de iguales características que los anteriores, concluye que no se ha mostrado ningún beneficio con el uso de esta terapia. ${ }^{(26)}$

\section{- Tratamiento combinado}

Un estudio aleatorizado doble ciego y controlado con placebo que estudio una combinación de baclofeno, naltrexona y sorbitol denominado PTX3003 con las siguientes: dosis baja (0,6 mg baclofeno, 0,07 mg de naltrexona y $21 \mathrm{mg}$ sorbitol), dosis intermedia (1,2 mg baclofeno, 0,14 mg de naltrexona y $42 \mathrm{mg}$ de sorbitol) o una dosis alta (6 mg de baclofeno, 0,7 mg de naltrexona y $210 \mathrm{mg}$ de sorbitol) de PXT3003 en pacientes con CMT1A, para este estudio utilizaron una muestra de 80 pacientes con dosis crecientes de la combinación de estos fármacos, este estudio se realizó por el lapso de un año y concluyo que el fármaco en altas dosis presentó una mejoría más allá de la estabilización de la enfermedad de un 8 \%. ${ }^{(27)}$

\section{- Medicamentos contraindicados}

Se debe tener en cuenta que ciertos fármacos se consideran tóxicos para en sistema nervioso periférico por lo que se recomienda limitar su uso, cuyos farmacos son: clorafenicol, amiodarona, alcohol, dapsone, disulfuro, izoniazida, hidralazina, megadosis de vitamina A, D, B6; metronidazol, nitrofurantoína, oxido nitroso, penicilina, cisplatino, vincristina entre otros. ${ }^{(9)}$

\section{Complicaciones}

Las complicaciones son variables y dependen de las comorbilidades que presente el paciente, entre las más comunes se encuentran:

- Osteoartritis: pueden afectarse las articulaciones de los tobillos, rodillas o caderas requiriendo reemplazos quirúrgicos articulares.

- Dolor neuropático característico (corriente eléctrica o fulgurante), se puede acompañar de calambres musculares y de síndrome de piernas inquietas. Para el manejo se debe realizar en conjunto con especialistas en manejo del dolor o también se puede utilizar fármacos como: gabapentina, pregabalina, duloxetina, amitriptilina, parches de lidocaína tópicos (dolor localizado) y los analgésicos opioides que deben ser usados con precaución. (3)

\section{Pronóstico}

La ECMT es una condición progresiva, tiene un ritmo de deterioro de aproximadamente 0,7 puntos por año en la escala CMTNSv2, lo que lleva a una diferencia significativa en el curso de la enfermedad del paciente cada 2 años aproximadamente. ${ }^{(3)}$

\section{Seguimiento}

Se recomienda hacer seguimiento cada 1 ○ 2 años para evaluar la progresión de los síntomas, esta evaluación debe incluir un examen físico neurológico completo, estudios de conducción nerviosa, consejo genético y ajustes en las medidas de rehabilitación, además un control anual para descartar la presencia de diabetes que puede exacerbar los síntomas del paciente, También se recomienda evaluar la relación daño-beneficio si el paciente necesita utilizar fármacos neurotóxicos, por el riesgo de padecer parálisis aguda. ${ }^{(4)}$

\section{Criterios de Referencia y contrareferencia:}

\section{Referencia:}

-Ante sospecha diagnóstica para realización de estudios complementarios.

-Control electromiográfico y valoración de deterioro patológico de manera anual.

- Presencia de complicaciones o comorbilidades de manejo limitado en primer nivel

-Deterioro mayor a 0.7 en la puntuación en la escala CMTNSv2 en 6 meses.

- Valoración para terapéutica quirúrgica.

-Apoyo psicológico para aceptación de patología y discapacidad.

- Valoración de grado de discapacidad.

-Manejo de rehabilitación y fisioterapia.

\section{Contrareferencia:}

- Una vez realizado el diagnóstico definitivo se complementará el manejo con una atención en primer nivel, con valoraciones clínicas, detección de progresión, complicaciones, comorbilidades, manejo sintomático y educacional con un periodo mínimo trimestral.

\section{Conclusiones}

-En nuestro país no se cuenta con la información epidemiológica exacta de los casos de ECMT, por lo que se considera una enfermedad infradiagnosticada.

El diagnóstico se realiza en base a los antecedentes familiares, clínica y estudios fisiológicos, las pruebas genéticas son fundamentales para obtener un diagnóstico específico y así poder brindar consejería familiar éstas pruebas presentan un alto costo por lo que se debe solicitar únicamente la evaluación de genes en sospecha.

-En Ecuador el Sistema de Salud está diseñado en 3 niveles de atención por lo que el paciente debe ser captado en primer nivel y derivado a un nivel superior para confirmar diagnóstico.

-Se concluye que la enfermedad de Charcot Marie Tooth, es una neuropatía hereditaria, progresiva que hasta la actualidad no se ha logrado identificar el tratamiento específico curativo de la enfermedad, por lo que su tratamiento es sintomático y de apoyo, enfocado en mejorar la calidad de vida.

El manejo y seguimiento debe ser llevado por un equipo multidisciplinario, realizándolo de 
manera trimestral e integral del paciente y su familia o cuidadores en un primer nivel de atención. El manejo de complicaciones, consulta quirúrgica para deformidad, apoyo psicológico y rehabilitación en un segundo nivel.

\section{Agradecimientos}

-A la Dra. Diana Martínez por su dirección en la estructuración de la presente investigación.

\section{Referencias}

1. Parsons T. Charcot-Marie-Tooth and Other Hereditary Motor and Sensory Neuropathies. Medscape[Online]: Nicholas Lorenzo; 22 de mayo 2018 [cited 2018 julio 18]. Available from: http://emedicine.medscape.com/ article/1173104-overview\#a0101.

2. Cruce T. Neuropatías hereditarias primarias motoras y sensoriales, incluyendo enfermedad de Charcot-Marie-Tooth.UpToDate. Online].; 2014 [cited 2015 enero 22. Available from: http://www.uptodate.com/ contents/hereditary-primary-motorsensoryneuropathies-including-charcot-marietoothdisease? source=search result\& search= charcot+marie\&selectedTitle $=1 \sim 43$.

3. Siskind C, Lewis R. Charcot Marie Tooth. BMJ. [Online]; 2014 [updated marzo 2018, cited 2015 enero 22]. Available from: http://bestpractice. bmj.com/best-practice/monograph/1 164.html.

4. Berciano J. Guidelines for molecular diagnosis of Charcot-Marie-Tooth disease. Elsevier. 2012 abril; [enero 2017] 27(3) : 169-178. Available from: http://www.elsevier.es/es-revistalable from: http://www.elsevier.es/es-revistapaciente-con-S0213485311002271

5. INEC. SISTEMA INTEGRADO DE CONSULTAS (redatam). [Online].; 2013 [cited 201709 23. Available from: http://redatam.inec.gob.ec/cgibin/ RpWebEngine.exe/PortalAction? \&MODE=MAIN \&BASE=VITAL2013\&MAIN=WebServerMain.inl.

6. Tazir M, Hamadouche T, Novioua S, Mathis S, Vallat JM. Hereditary motor and sensory neu ropathies or Charcot-Marie-Tooth diseases. JNS. [Online] Algeria 2014 [cited 2016 octubre 25; 347(1-2): 14-22 Available from: https:// www.ncbi.nlm.nih.gov/pubmed/25454638.

7. Errando C. Orfhananesthesia, Recomendaciones para anestesia en pacientes afectados por Charcot Marie Tooth. Orphanet [Online]. España; 2014 [cited 2017 Septiembre 30. Available from: https://www.orpha.net/data/ patho/Pro/es/Charcot_Marie_Tooth_ES.pdf.

8. Nascimento OJ. The broad clinical spectrum of hereditary neuropathy with liability to pressure palsy (HNPP). Scielo.[Online] 2016 January; [cited 2017 Septiembre 30] 74(2). http://www.scielo.br/scielo.php? script=sci arttext\&pid=S0004-282X2016000200002

9. LG II INR. Manual de Guía Clínica de Enfermedades neuromusculares (neuropatía periférica hereditaria Charcot Marie Tooth). LG II ferica hereditaria Charcot Marie Tooth). LG II 1-21. Available from: http://iso9001.inr.gob. mx/Descargas/iso/doc/MG-SMR-15.pdf.

10. Bird TD. Charcot-Marie-Tooth Neuropathy Type 1, genereviews. [Online].; Adam MP Seattle (WA) 2015 [cited 2017 Octubre 30]. Available from: https://www.ncbi.nlm.nih. gov/books/NBK1205/.

11. Barisic N, et al. Charcot-Marie-Tooth Disease: A Clinico-geneticConfrontation. Annals of Human Genetics.[Online]. Thomas, Croacia, 2008; [cited 2017 Octubre 30], 72/416 -441). https://onlinelibrary.wiley.com/doi/ full/10.1111/j.1469-1809.2007.00412.x

2. Cortes $\mathrm{H}$, et al. Un vistazo a la enfermedad de Charcot-Marie-Tooth. Investigacion en Discapacidad. [Online].; 2015 [cited 2017 octubre 08] 1 (2): 77-82. Available from: http:// www.medigraphic.com/pdfs/invdis/ir-2012/ ir 122e.pdf

13. Aguilar L, et al. Enfermedad de CharcotMarie-Tooth: actualidad y perspectivas. Arch Neurocien. [Online]: 2012 Junio: 17(2). 110Neurocien. [Online].; 2012 Junio; 17(2). 110-
118. Available from: http://www.medigraphic. 118. Available from: http://www.medigraph
com/pdfs/arcneu/ane-2012/anel22f.pdf

14. Lewis R. Polineuropatia inflamatoria desmielinizante crónica. [Online] UpToDate; Shefner J 2014 [cited 2015 enero 20]. Available from: https://www.uptodate.com/ contents/chronic-inflammatory-demyelicontents/chronic-inflammatory-demyelinating-polyneuropathy-etiology-clinical-
features-and-diagnosis? search=charcot\%20 marie\%20tooth\%20adultos\&source=searchresult\&selectedTitle =6 4 1 \&usage type $=$ default \&display_rank $=6$

15. Feldman EL. Clinical manifestations and diagnosis of diabetic polyneuropathy. [Onine] UpToDate; Shefner J 2017 [cited 2017 septiembre 30]. Available from: https:// www.uptodate.com/contents/clinicalmanifestations-and-diagnosis-of-diabetic-polyneuropathy? search=Clinical\%20 manifestations\% 20 and \% 20 diagnosis\%20 of\%20diabetic\%20polyneuropathy \&source $=$ search result\&selectedTitle $=1 \sim 1$ 50\&usage_type=default\&display_rank $=1$

16. Kang P. Información general de las neuropatías periféricas adquiridas en los niños.. [Online]. UpToDate; Shefner J 2014 [update 2018 cited 2015 enero 20. Available from: https://www.uptodate.com/contents/ overview-of-acquired-peripheral-neuropathies-in-children? search=NEUROPATIAS\%20 Pathies-in-children? search=NEUR\%20ADQUIRIDAS\%20EN\%20 PERIFICAS\%20ADQUIRID AS\%20EN\% 20
NI:OS\&source=search_result\&selectedTit $l e=1 \sim 150 \&$ usage_type $=$ default\&display rank=1 \# H438968953

7. Opal P, et. al. Paraplejía espástica hereditaria. [Online] UpToDate: Pathereditaria. [Online] UpToDate; Pat-

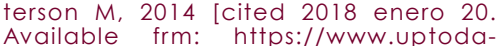
Available frm: https://www.uptodaparaplegia? search = paraplejia\% 20 e s p \% C $3 \%$ A l s $t$ i c a $\% 20$ hereditaria\&source =search result\&selectedTitle=1 15\&usage type=default\&display_rank=1

18. Murfhy S. Reliability of the CMT neuropathy score (second version) in Charcot-MarieTooth disease. [Online] $\mathrm{NIH}$; London, Peripher J, 2015 [cited 2015 enero 20]. Available from: http://www.ncbi.nlm.nih.gov/pmc/articles/ PMC3754828/table/T1/.

19. Cordeiro J, et. al. La enfermedad de CharcotMarie-Tooth, indicadores psiquiátricos y calidad de vida. ASN [Online]; 2014 [cited 2015 enero 22.] 6(3):185-92. Available from: http:// www.ncbi.nlm.nih.gov/pubmed/24654889.

20. Pareyson D, et al. Ascorbic acid in CharcotMarie-Tooth disease type 1A (CMT-Triaal and CMt-trauk): a double-blind randomised trial. Lancet Neurology. The Lancet Neurology [Online]; 2011 [cited 2015 octubre 8] 10(4) 320-328. Available from: https://www.thelancet.com/journals/laneur/article/PIIS 14744422(1 1)70025-4/fulltext

21. Dimitrova $E$, et al. The Role of Rehabilitation in the Management of Patients with CharcotMarie-Tooth Disease: Report of Two Cases, Open Access Macedonian Journal of Medical Sciences. [Online]; 2016 [cited 2016 octubre 28] 4 (3) 443-448 Available from: https://www.id-press.eu/mjms/article/view/ oamjms.2016.079/1094

22. Vita G, et. al. Sport activity in Charcot-MarieTooth disease: A case study of a Paralympic swimmer, Neuromuscular Disorders. [Online]; 2016 [cited 2016 octubre 28]. 26(9) 614-618 Available from: https://www.ncbi.nlm.nih. gov/pubmed/27460291.

23. Djordjevic D, et. al. Effects of Self-Selected Exercise on Strength in Charcot-Marie-Tooth Disease Subtypes. Can J Neurol Sci.. [Online].;
2017 [cited 2017 septiembre 30]. 44(5): 572576 Available from: https://www.ncbi.nlm.nih. gov/pubmed/28669366

24. Pareyson D, et. al. Efecto del ácido ascórbico en pacientes con enfermedad de CharcotMarie-Tooth tipo 1 A: un ensayo clínico multicéntrico, aleatorizado, doble ciego, conticéntrico, aleatorizado, doble ciego, con-
trolado con placebo. Lancet Neuro. [Online] 2011 july[cited 2015 enero 22] 10(4) 320-328.

25. Gess B, Young P. El ácido ascórbico y transportadores de sodio dependiende de la vitamina $C$ en el sistema nervioso periferico, ARS [Online]; 2013 [cited 2015 enero 22] 19(17) 2105- 2014. Available from: http://www.ncbi. nlm.nih.gov/pubmed/23642070.

26. James $\mathrm{P}$, et. al. Recomendaciones actuales para el tratamiento de la hipertensión JNC8. JAMA. [Online].; 2014 [cited 2015 enero 22]. 311 (5):507-520 Available from: https:// jamanetwork.com/journals/jama/fullarticle/1791497

27. Attarian S, et. al. An exploratory randomised double-blind and placebo-controlled phase 2 study of a combination of baclofen, naltrexone and sorbitol (PXT3003) in patients with Charcot-Marie-Tooth disease type $1 \mathrm{~A}$ Orphanet. [Online]; Francia, 2014 [cited 2016 octubre 29]. Available from: https://www. ncbi.nlm.nih.gov/pmc/articles/PMC4311411.

28. Berciano J. et. al. Guia para el diagnostico molecular de Charcot-Marie- Tooth. Neurologia. [Online]: 2012 [cited 2017 octubre 08]. 22(3): 168-178. Available from: http:// www.elsevier.es/en-revista-neurologiaenglish-edition--495-articulo-guidelinesfor-molecular-diagnosis-charcot-mariefor-molecular-diagnosis-c
tooth-S2173580812000417. 